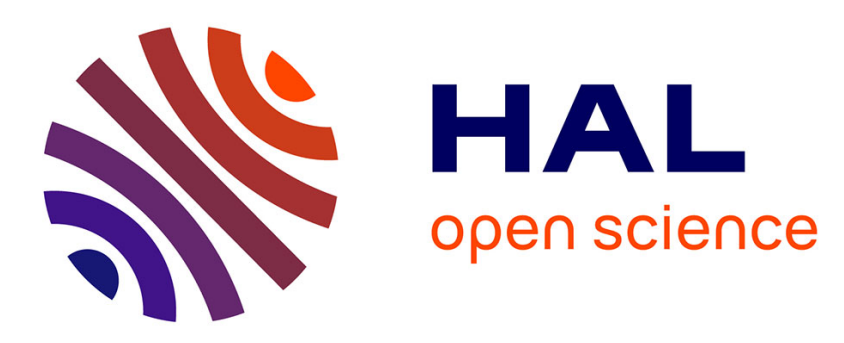

\title{
On the discretization of contact problems in elastodynamics
}

\author{
Houari Boumediène Khenous, Patrick Laborde, Yves Renard
}

\section{To cite this version:}

Houari Boumediène Khenous, Patrick Laborde, Yves Renard. On the discretization of contact problems in elastodynamics. Wriggers P; Nackenhorst U. Analysis and Simulation of Contact Problems, 27, Springer, pp.31-38, 2006, Lecture Notes in Applied and Computational Mechanics, 9783540317616. 10.1007/3-540-31761-9_4. hal-01725015

\section{HAL Id: hal-01725015 \\ https://hal.science/hal-01725015}

Submitted on 7 Mar 2018

HAL is a multi-disciplinary open access archive for the deposit and dissemination of scientific research documents, whether they are published or not. The documents may come from teaching and research institutions in France or abroad, or from public or private research centers.
L'archive ouverte pluridisciplinaire HAL, est destinée au dépôt et à la diffusion de documents scientifiques de niveau recherche, publiés ou non, émanant des établissements d'enseignement et de recherche français ou étrangers, des laboratoires publics ou privés. 


\title{
On the discretization of contact problems in elastodynamics
}

\author{
H.B. Khenous ${ }^{1}$, P. Laborde ${ }^{2}$, and Y. Renard ${ }^{1}$ \\ 1 INSA, Département de Génie Mathématique, 135 avenue de Rangueil, 31077 \\ Toulouse cedex, France, \\ khenous@insa-toulouse.fr, renard@insa-toulouse.fr \\ 2 Laboratoire MIP, 118 route de Narbonne, 31062 Toulouse cedex, France, \\ laborde@mip.ups-tlse.fr
}

In this work, we will presente a comparison of two formulation for the discretization of elastodynamic contact problems. The first approach consists on a midpoint scheme and a contact condition expressed in terms of velocity. This approach gives an energy conserving scheme. The second one we propose is a new distribution of the solid mass. The problem expressed with the new mass matrix is well posed, energy conserving and has a lipschitz solution. Finally, some numerical results are presented.

\section{Introduction}

The contact problem attracts considerable attention from the computational mechanics community, due in large part to its highly non-linear and discontinous nature. Indeed, engineering analysts charged with solving such problems will attest merely achieving convergence of non-linear solution schemes can be difficult under many circumstances. This difficulties stem primary from the fact that elastodynamic contact problems are not well posed [8].

For purely contact elastodynamic problems (hyperbolic problems), as far as we now, existence result has been proved in a scalar two dimensional case by Lebeau-Schatzman [4], Kim [3] and in the vector case with a modified contact law by Renard-Paumier [9]. It seems that no energy conserving result has been proved. In this work, we will present two numerical energy conserving strategies for elastodynamic contact problems.

The plan of the paper is as follows. Section 2 we propose a semi-discretization using a finite element method, give the equivalent contact condition and the time integration scheme used and discuss the energy conserving of the algorithm. Section 3 we consider an equivalent mass matrix so that the equivalent finite element problem is energy conserving. Some numerical tests are presented in Section 4 to demonstrate performance of the two approaches. 


\section{Method with the equivalent contact condition ECC}

In elastodynamics, the classical contact condition is not sufficient to deal with the problem correctly. Of course, in the enrergy analysis of any time integration scheme for contact problems, we remark that a sort of contact condition in terms of velocity appears. The stake is how to define this contact condition which can replace the classical one. Then, many authors try to give a new formulation for the contact condition: Laursen-Love [6], Moreau [7] and obtain an interesting results. In this section, we deal with such condition.

We assume a vanishing initial gap between the structure and the rigid foundation and we denote $u^{0}, u^{1}$ and $T$ the given initial displacement, initial velocity and time simulation respectively.

The space semi-discretization of the elastodynamic contact problem with nodal contact condition is defined for a Lagrange finite element method as follows. Find $u:[0, T] \longrightarrow \mathbb{R}^{d}$ such that

$$
\left\{\begin{array}{l}
M \ddot{u}+K u=f+\sum_{i \in I_{C}} \lambda_{N}^{i} N_{i}, \quad \text { in }[0, T] \times \mathbb{R}^{d}, \\
\lambda_{N}^{i} \leq 0, u \cdot N_{i} \leq 0, \lambda_{N}^{i}\left(u \cdot N_{i}\right)=0, \forall i \in I_{C} \\
u(0)=u^{0}, \dot{u}(0)=u^{1}
\end{array}\right.
$$

where $d$ is the number of degrees of freedom for the displacement $u$, the notations $M, K, f$ stend for the mass matrix, the stiffness matrix and the given force densities respectively, $I_{C}$ is the set of the contact boundary indices. On each node $i \in I_{C}$, we denote $\lambda_{N}^{i}$ and $N_{i}$ the normal stress and the outward unit normal respectively.

Problem (1) is not well posed (see [7]). However, uniqueness can be recovered, for rigid bodies, by introducing an impact law with a restitution coefficient. This seems not to be completly satisfying for deformable bodies because whatever the restitution coefficient value, the system tends to a global restitution of energy when the mesh parameter goes to zero (more details will be presented in [1]).

We replace the classical Signorini condition in Problem (1) by the following condition

$$
\left\{\begin{array}{l}
u \cdot N_{i}<0 \Longrightarrow \lambda_{N}^{i}=0, \\
u \cdot N_{i} \geq 0 \Longrightarrow \dot{u} \cdot N_{i} \leq 0, \quad \lambda_{N}^{i} \leq 0, \quad \lambda_{N}^{i}\left(\dot{u} \cdot N_{i}\right)=0 .
\end{array}\right.
$$

The expression (2) in terms of velocity is very close to the one introduced in [7] and corresponds to the one introduced in [5]. The velocity is to be understood as a right derivative and the second condition implies in fact the non-interpenetration.

We discretize the elastodynamic part in Problem (1) by a midpoint scheme as follows: 


$$
\left\{\begin{array}{l}
u^{0} \text { et } v^{0} \text { donnés, } \\
u^{1}=u^{0}+\Delta t v^{0}+\Delta t z(\Delta t) / \lim _{\Delta t} z(\Delta t)=0, \\
M\left(\frac{u^{n+1}-2 u^{n}+u^{n-1}}{\Delta t^{2}}\right)+K\left(\frac{u^{n+1}+2 u^{n}+u^{n-1}}{4}\right) \\
=f+\sum_{i \in I_{C}} \lambda_{N}^{i, n} N_{i}, \forall n \geq 1,
\end{array}\right.
$$

where $\Delta t$ is the time parameter. The contact condition (2) is approximated using a central difference scheme:

$$
\left\{\begin{array}{l}
u^{n} \cdot N_{i}<0 \Longrightarrow \lambda_{N}^{i, n}=0, \\
u^{n} \cdot N_{i} \geq 0 \Longrightarrow\left\{\begin{array}{l}
\frac{\left(u^{n+1}-u^{n-1}\right) \cdot N_{i}}{2 \Delta t} \leq 0, \quad \lambda_{N}^{i, n} \leq 0, \\
\lambda_{N}^{i, n}\left(\frac{\left(u^{n+1}-u^{n-1}\right) \cdot N_{i}}{2 \Delta t}\right)=0 .
\end{array}\right.
\end{array}\right.
$$

Theorem 1. The stability of scheme (3)-(4) is ensured by the fact that the discrete energy

$$
J(u, v)=\frac{1}{2}\left\langle M v, v>+\frac{1}{2}\langle K u, u>-<f, u>\right.
$$

is conserved in the following sense

$$
\Delta J=J\left(u^{n+\frac{1}{2}}, v^{n+\frac{1}{2}}\right)-J\left(u^{n-\frac{1}{2}}, v^{n-\frac{1}{2}}\right)=0,
$$

with $u^{n+\frac{1}{2}}=\frac{u^{n+1}+u^{n}}{2}, \quad v^{n+\frac{1}{2}}=\frac{u^{n+1}-u^{n}}{\Delta t}$.

Proof. One has

$$
\begin{aligned}
\Delta J & =\frac{1}{2}<M\left(v^{n+\frac{1}{2}}+v^{n-\frac{1}{2}}\right), v^{n+\frac{1}{2}}-v^{n-\frac{1}{2}}> \\
& +\frac{1}{2}<K\left(u^{n+\frac{1}{2}}+u^{n-\frac{1}{2}}\right), u^{n+\frac{1}{2}}-u^{n-\frac{1}{2}}>-<f, u^{n+\frac{1}{2}}-u^{n-\frac{1}{2}}>.
\end{aligned}
$$

Then, using the definition of $u^{n+\frac{1}{2}}$ and $v^{n+\frac{1}{2}}$, we obtain:

$$
\begin{aligned}
\Delta J & =\frac{1}{2 \Delta t^{2}}<M\left(u^{n+1}-2 u^{n}+u^{n-1}\right), u^{n+1}-u^{n-1}> \\
& +\frac{1}{8}<K\left(u^{n+1}+2 u^{n}+u^{n-1}\right), u^{n+1}-u^{n-1}>-\frac{1}{2}<f, u^{n+1}-u^{n-1}>.
\end{aligned}
$$

Hence, from (3):

$$
\Delta J=\frac{1}{2}<\sum_{i \in I_{C}} \lambda_{N}^{i, n} N_{i}, u^{n+1}-u^{n-1}>=\Delta t \sum_{i \in I_{C}} \lambda_{N}^{i, n}\left(\frac{u^{n+1} \cdot N_{i}-u^{n-1} \cdot N_{i}}{2 \Delta t}\right) .
$$

Finally, (4) leads to $\Delta J=0$. Then, the first approach is energy conserving. 


\section{Remark.}

- The major difficulty with scheme proposed in [5] is that the contact condition (2) is discretized using a central diffence scheme.

- In this section, we proved conservation energy of the discretized contact elastodynamic problem using an appropriate scheme and a choice of contact condition in terms of velocity. But this condition, as it was approximated, allows some interpenetration. Then, we opt for an other approach which can take in acounte our no-iterpenetration property and conserve energy. This is what we are going to do in the following section.

\section{Equivalent mass matrix method (EMM)}

The non-well-posedness of Problem (1) comes from the fact that the nodes on the contact boundary have their own inertia. This leads to instabilities especially for energy conserving schemes. We propose here to introduce a new distribution of the mass with the same total mass, center of gravity and inertia momenta. This distribution of the mass is done so that there is no inertia for the contact nodes (similarly to what happens in the continuous case). We refer the reader to [1] for further details. We assume that the modified mass matrix is still denoted $M$ such that $N_{i}^{T} M N_{j}=0, \forall i, j \in I_{C}$. Then, the two following results hold.

Theorem 2. Problem (1) with the equivalent mass matrix is well posed and has a Lipschitz continuous solution.

Proof. If we order the degrees of freedom such that the last ones are the nodes on the contact boundary, we can split each matrix and vector in interior part and contact boundary part as follows:

$$
M=\left(\begin{array}{cc}
\bar{M} & 0 \\
0 & 0
\end{array}\right), K=\left(\begin{array}{cc}
\bar{K} & C^{T} \\
C & D
\end{array}\right), N_{i}=\left(\begin{array}{c}
0 \\
\tilde{N}_{i}
\end{array}\right) \text { and } u=\left(\begin{array}{c}
\bar{u} \\
\tilde{u}
\end{array}\right) .
$$

Then, Problem (1) becomes:

$$
\left\{\begin{array}{l}
\bar{M} \ddot{\bar{u}}+\bar{K} \bar{u}=f+C^{T} \tilde{u} \\
D \tilde{u}+C \bar{u}=\sum_{i \in I_{C}} \lambda_{N}^{i} \tilde{N}_{i} \\
\lambda_{N}^{i} \leq 0, u \cdot N_{i} \leq 0, \lambda_{N}^{i}\left(u \cdot N_{i}\right)=0, \forall i \in I_{C} \\
u(0)=u^{0}, \dot{u}(0)=u^{1}
\end{array}\right.
$$

The second equation together with the contact condition uniquely define $\tilde{u}$ as soon as $\bar{u}$ is given. Furthermore, $\tilde{u}$ depends Lipschitz continuously on $\bar{u}$. The first equation is a lipschitz ordinary differential equation. 

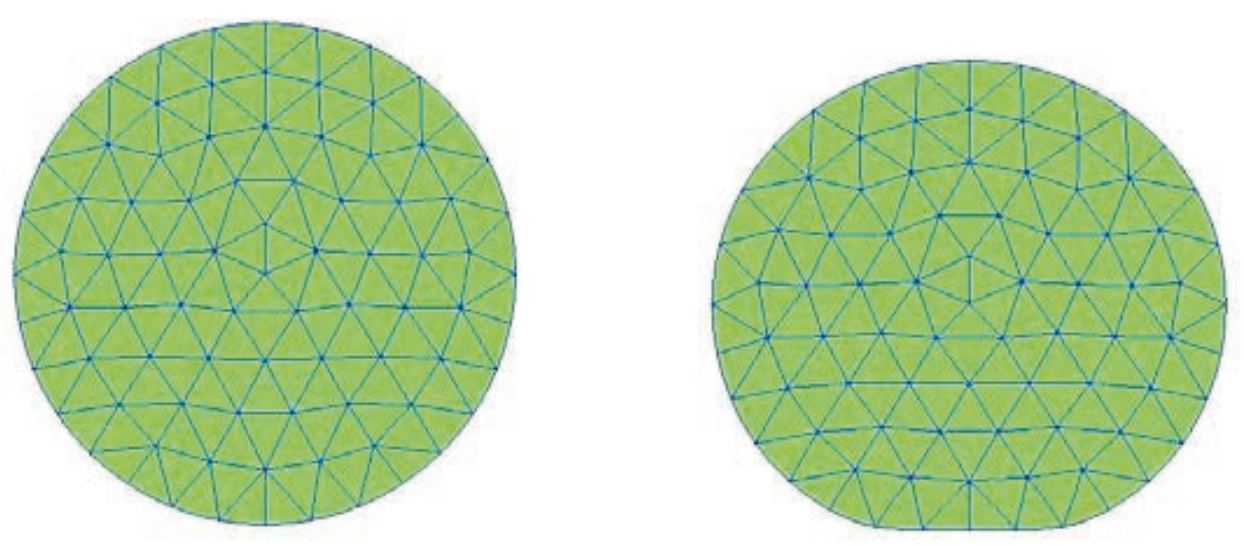

Fig. 1. A disc before and during the first contact.

Theorem 3. Problem (1) with the equivalent mass matrix is energy conserving.

We refer to [1] for a completely proof. This result comes from the fact that $\bar{u}_{i}, \tilde{u}_{i}$, and $\lambda_{N}^{i}$ satisfy $\bar{u}_{i} \in \mathcal{C}^{2}([0, T], \mathbb{R}), \tilde{u}_{i} \in W^{1, \infty}([0, T], \mathbb{R})$ and $\lambda_{N}^{i} \in W^{1, \infty}([0, T], \mathbb{R})$.

\section{Numerical results}

In this section, we study the dynamic contact of an elastic disc (see Fig. 1) the properties of which are summerized in Tab. 1 . We denote $A$ the lowest point of the disc (the first point which will be in contact).

Table 1. Characteristics of the elastic disc and the resolution method

\begin{tabular}{|c|c|c|c|}
\hline Disc property & Value & Property of the resolution method & Value \\
\hline$\rho$, diameter & $610^{-6} \mathrm{~kg} / \mathrm{cm}^{3}, 20 \mathrm{~cm}$ & Time step & $10^{-4} \mathrm{~s}$ \\
\hline Lamé coefficients & $\lambda=10 \mathrm{GP}, \mu=5 \mathrm{GP}$ & Simulation time & $0.3 \mathrm{~s}$ \\
\hline$u^{0}, v^{0}$ & $1 \mathrm{~cm},-100 \mathrm{~cm} / \mathrm{s}$ & Mesh parameter & $\simeq 2 \mathrm{~cm}$ \\
\hline
\end{tabular}

The results of simulations for the midpoint scheme with the equivalent contact condition ECC are presented in Fig. 2. The energy is indeed constant, however the normal stress in point $A$ is very noisy and inexploitable. Concerning Problem (1) with a modified mass matrix, the simulations are done using a Newmark scheme with $\beta=\gamma=0.5$. Fig. 3 shows that the normal stress in $A$ for the second approach is more regular than for the first one. Moreover, there is very small fluctuations in the energy evolution which is quasi-conserved. 

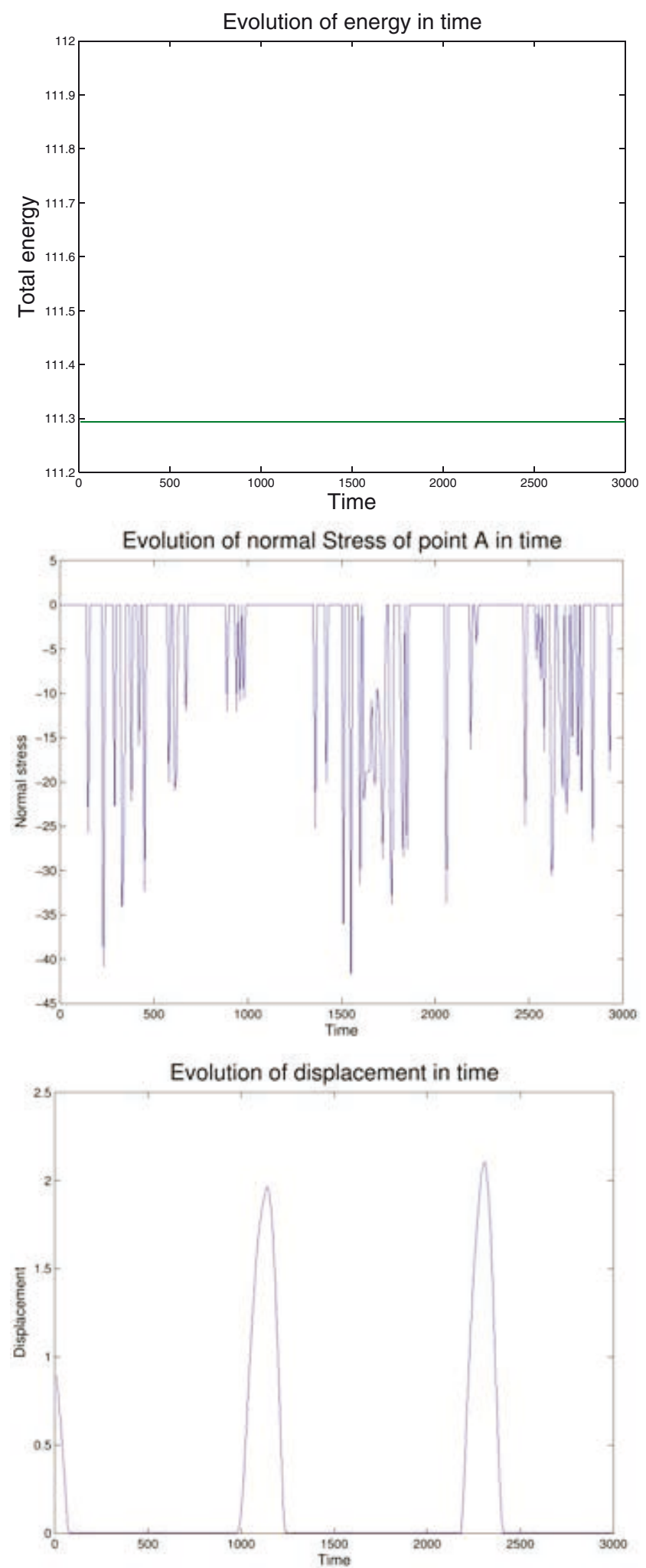

Fig. 2. Energy, normal stress and displacement evolution for the midpoint scheme with ECC condition. 

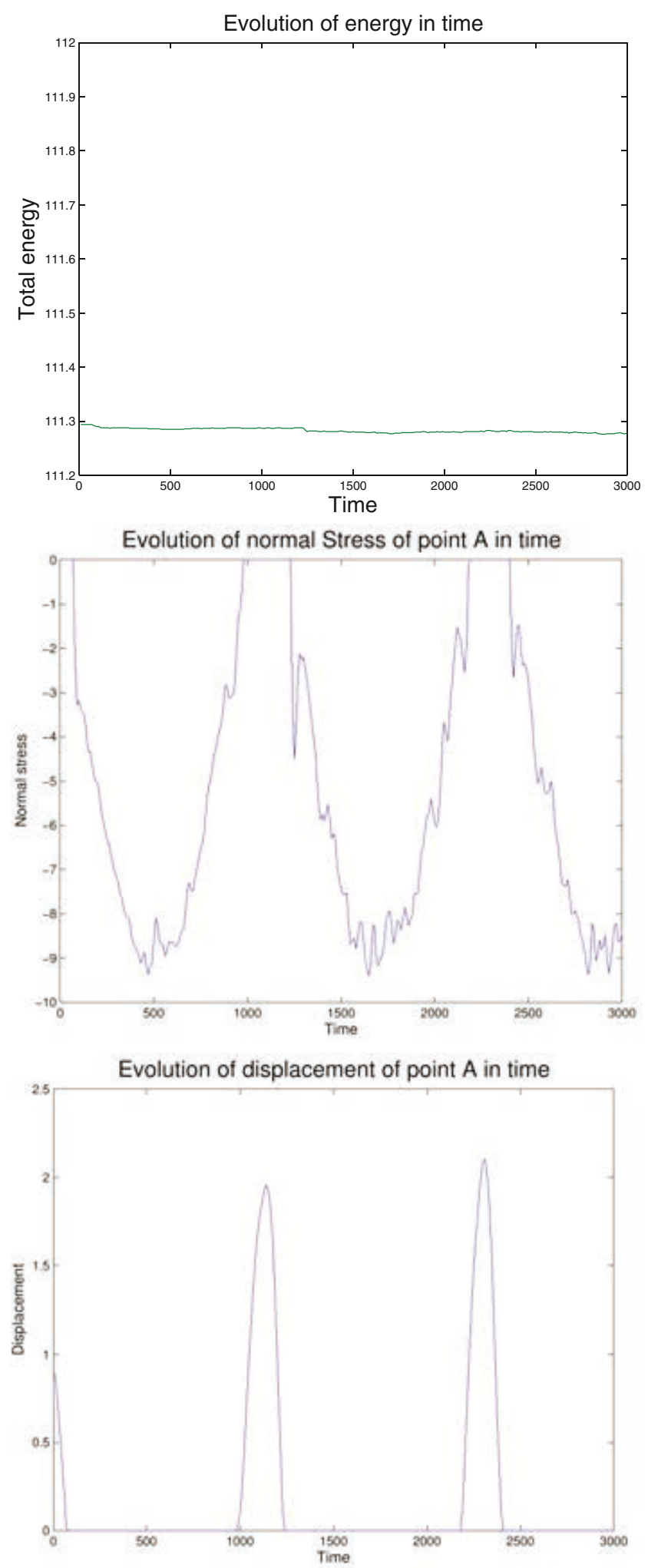

Fig. 3. Energy, normal stress and displacement evolution for the Newmark scheme with EMM method. 


\section{Concluding remarks}

In this work, we proved the stability of elastodynamic contact problem using an appropriate time integration scheme. The first approach we presented ensure conservation of energy but allows a small interpenetration and the computed normal stress is badely approximated. It could be interesting to see that this scheme is well adapted for rigid bodies. The second approach is very simple to implement and gives a good approximation of normal stress. For both approaches, adding a Coulomb friction condition is not a difficulty from the stability view point. However, this condition could be badly approximated for the first approach because it depends on the normal stress.

\section{References}

1. H.B. Khenous, $\mathrm{PhD}$ thesis, INSA of Toulouse, in preparation.

2. H.B. Khenous, Y. Renard \& J. Pommier, Hybrid discretization of the Signorini problem with Coulomb friction. Theoretical aspects and comparison of some numerical solvers, to appear.

3. J.U. Kim, A boundary thin obstacle problem for a wave equation, Com. part. diff. eqs., 1989, 14 (8\&9), 1011-1026.

4. G. Lebeau \& M. Schatzman, A wave problem in a half-space with a unilateral constraint at the boundary, J. diff. eqs., 1984, 55, 309-361.

5. T.A. Laursen \& V. Chawla, Design of energy conserving algorithms for frictionless dynamic contact problems, Int. J. Num. Meth. Engrg, Vol. 40, 1997, 863-886.

6. T.A. Laursen \& G.R. Love, Improved implicit integrators for transient impact problems-geometric admissibility within the conserving framework, Int. J. Num. Meth. Eng, 2002, 53, 245-274.

7. J.J. Moreau, Numerical aspects of the sweepig process, Comp. Meth. Appl. Mech. Engrg., 1999, 177, 329-349.

8. L. Paoli, Time discretization of vibro-impact, Phil. Trans. R. Soc. Lond. A., 2001, 359, 2405-2428.

9. Paumier \& Y. Renard, Surface perturbation of an elastodynamic contact problem wih friction, European Journal of Applied Mathematics, vol. 14, 2003, 465-483. 